\title{
Estrategias educativas y de evaluación en la formación de médicos especialistas
}

\author{
Verónica Alejandra Gaona-Flores', Luz Arcelia Campos-Navarro², Jesús Arenas-Osuna³ \\ y Enrique Alcalá-Martínez ${ }^{1}$ \\ ${ }^{1}$ Hospital de Infectología; ${ }^{2}$ Hospital General; ${ }^{3}$ Hospital de Especialidades. Centro Médico Nacional La Raza, Ciudad de México, México
}

\begin{abstract}
Resumen
Antecedentes: Las estrategias didácticas se han definido como procedimientos, medios o recursos que el docente utiliza para promover el aprendizaje significativo. Objetivo: Identificar las estrategias de enseñanza y de evaluación que utiliza el profesor con residentes en hospitales de tercer nivel de atención médica. Método: Estudio transversal, con profesores de diversas especialidades, en el Centro Médico Nacional La Raza. Se aplicó un cuestionario que valora las estrategias utilizadas en la enseñanza y la evaluación. Resultados: En una muestra de 90 profesores, 35 especialidades médicas con profesores ayudantes, adjuntos y titulares, las actividades de enseñanza que más realizan son organizar a los alumnos a exponer temas, seguido de realizar preguntas sobre temas vistos. Respecto a las estrategias educativas, la más frecuente fue análisis de casos y aprendizaje basado en problemas. Para evaluación del desempeño teórico, los métodos más utilizados fueron participación en clase, exposición de temas y exámenes. Conclusiones: las actividades de enseñanza están basadas en la exposición de temas por los médicos residentes. La estrategia educativa más utilizada fue llevar a cabo análisis de casos clínicos, seguido por aprendizaje basado en problemas. La evaluación del desempeño en aspectos teóricos se realiza a partir de la participación en clase, la exposición de temas y exámenes.
\end{abstract}

PALABRAS CLAVE: Educación. Educación médica. Estrategias educativas. Evaluación del aprendizaje.

\begin{abstract}
Background: Teaching strategies have been defined as procedures, means or resources that teachers used to promote meaningful learning. Aim: Identify teaching strategies and evaluation used by the professor with residents in tertiary hospitals health care. Methods: This is a cross-sectional study conducted with full, associate and assistant professors of various medical specialties. A questionnaire was applied to evaluate the strategies used by professors to teach and evaluate students. Results: We included a sample of 90 professors in 35 medical specialties. The most frequent teaching activities were: organizing students to develop presentations on specific subjects, followed by asking questions on previously reviewed subjects, In terms of the strategies employed, the most frequent "always" option was applied to case analyses. The most frequent methods used for the evaluation of theoretical knowledge were: participation in class, topic presentation and exams. Conclusions: Teaching activities were primarily based on the presentation of specific topics by the residents. The most commonly used educational strategies were clinical case analyses followed by problem-based learning and the use of illustrations. Evaluation of the residents' performance in theory knowledge, hinged on class participation, presentation of assigned topics and exams.
\end{abstract}

KEY WORDS: Education. Medical education. Educational strategies. Learning assessment.

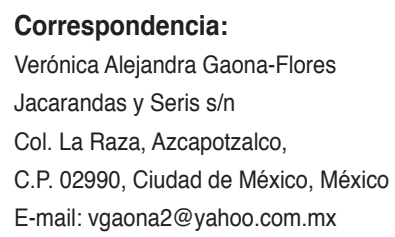

Fecha de aceptación en versión modificada: 09-05-2016

Fecha de aceptación: 09-05-2016

DOI:10.24875/GMM.17002539
Gac Med Mex. 2017;153:503-509

Contents available at PubMed www.gacetamedicademexico.com 


\section{Introducción}

La enseñanza clínica involucra directamente a los pacientes y constituye el aspecto medular de la educación en el ámbito de la salud. El proceso de enseñanza-aprendizaje en la educación médica es de suma importancia; es a partir de ello que el médico en formación obtiene capacidades para resolver los problemas que le plantea la atención médica'.

Así, este aprendizaje se enfoca en problemas reales en el contexto del ejercicio profesional, y de esta forma los estudiantes se motivan por su participación en la resolución de estos problemas. La enseñanza típica involucra la supervisión del estudiante en formación por parte de un clínico con mayor experiencia; generalmente vincula al residente de mayor jerarquía 0 al profesor ayudante, lo que da lugar a una amplia variedad de estilos de enseñanza. Un aspecto importante a destacar en este escenario es que una parte importante del aprendizaje se obtiene desde el ejemplo ofrecido por los profesores de ese momento?

Ante las expectativas de los médicos residentes, que son múltiples, pareciera que el profesor eficaz es quien es capaz de satisfacer las necesidades académicas de los estudiantes. El desempeño del residente depende de condiciones que el profesor debe saber reconocer e identificar; algunos factores son los curriculares y los del estilo de aprendizaje del alumno. Se conceptualiza el estilo de enseñanza como el comportamiento que el docente exhibe en cada fase o momento de la actividad de enseñanza, que se fundamenta en actitudes personales que le son características, que han sido abstraídas de su experiencia académica y profesional, que no dependen de los contextos en los que se muestran y que pueden aumentar o disminuir los desacuerdos entre la enseñanza y el aprendizaje ${ }^{3}$. Estos elementos conforman un estilo de enseñanza y de aprendizaje, no podemos determinar solo un estilo de uno u otro; en general, es heterogéneo e incluso cambia en cada alumno, aula o situación clínica. Este comportamiento es dinámico, pues en la enseñanza implica conocer al alumno, su personalidad, su inteligencia, sus fortalezas o debilidades intelectuales y emocionales, los motivos que guían su aprendizaje, y ello permite al docente crear una atmosfera apropiada para el proceso de enseñanza y aprendizaje ${ }^{4}$.

No existe un estilo de enseñanza ideal y agradable para todos los estudiantes, puesto que las relaciones entre los estilos de enseñanza y el aprendizaje son complejas y existe una sustancial variabilidad individual.

Las estrategias didácticas se han definido como procedimientos, medios o recursos que el docente utiliza en forma reflexiva y flexible para promover el logro del aprendizaje significativo. Es importante identificar que la didáctica está condicionada por la especificidad del currículum propio de la especialidad ${ }^{5}$. En forma consecutiva en el proceso enseñanza-aprendizaje está la evaluación, entendida como una variable que sustenta, entre otros, los criterios propios del docente en la evaluación del aprendizaje, congruencia o retroalimentación académica ${ }^{6,7}$.

Se han realizado algunos estudios acerca de la relación entre el estilo de enseñanza del profesor y de aprendizaje de los alumnos. Destaca que cada alumno tiene su propio estilo de aprendizaje independiente del estilo de enseñanza que tenga el profesorado, y que no está directamente relacionado con el rendimiento del alumno, si bien se ha podido identificar que prefieren las metodologías docentes más centradas en el alumno ${ }^{8}$. Otra publicación identificó que se requiere fortalecer la actitud y la motivación para estudiar de los residentes, y con ello proporcionar cursos sobre técnicas de estudio9,10.

La evaluación es una estrategia didáctica que permite verificar el proceso educativo. En general se lleva a cabo mediante la observación y la interrelación del estudiante con el docente en la práctica clínica para posteriormente plasmar de manera cualitativa o cuantitativa el resultado obtenido; esto se muestra como un reto para el docente. Para interpretar la evaluación se deben definir las metas a cumplir por el estudiante y notificar los objetivos del curso para lograr el desempeño.

La evaluación diagnóstica, sumativa y formativa, es la evaluación formal que se debe llevar a cabo a través de formatos o instrumentos de evaluación, incluso con listas de cotejo que faciliten el registro de la información obtenida por cada estudiante en el ámbito de las competencias cognitivas, procedimentales y actitudinales ${ }^{11,12}$.

Bajo estos elementos surgió la pregunta de investigación para este estudio: ¿cuáles son las estrategias educativas y de evaluación con las que se forman los médicos especialistas en hospitales de tercer nivel de atención de un centro médico nacional? Así, nuestro objetivo fue identificar las estrategias de enseñanza y de evaluación que utiliza el profesor con residentes en los hospitales de tercer nivel de atención médica. 


\section{Método}

Estudio observacional, descriptivo, transversal, que se llevó a cabo en el Centro Médico Nacional La Raza, de enero a junio de 2015, con profesores titulares, adjuntos y ayudantes de diversas especialidades médicas que desearon participar; se les invitó de forma verbal, leyendo un guion de consentimiento informado para contestar un cuestionario sobre las estrategias educativas y de evaluación utilizadas con los residentes. El lugar de reunión para la aplicación del cuestionario fue el auditorio del hospital y les tomó aproximadamente diez minutos contestarlo.

El instrumento fue proporcionado por la autora de un estudio realizado en la Facultad de Odontología en la Universidad Nacional Autónoma de México. El resultado del análisis de consistencia interna con alfa de Cronbach fue de 0.880 , lo que determinó que el instrumento fuera confiable. Estuvo conformado por tres apartados que evalúan las estrategias utilizadas por el profesor en la enseñanza y la forma de evaluar a los alumnos. La discriminación de las respuestas fue a través de puntajes en una escala de Likert con cuatro opciones: nunca, casi nunca, casi siempre y siempre ${ }^{13}$.

\section{Análisis estadístico}

Se realizó estadística descriptiva con números absolutos y porcentajes.

\section{Aspectos éticos}

El estudio fue sometido al Comité Local de Investigación en Salud del Instituto Mexicano del Seguro Social (IMSS). Según los criterios del Reglamento de la Ley General de Salud en Materia de Investigación para la Salud, título segundo "De los aspectos éticos de la investigación en seres humanos», capítulo único, artículo 17, inciso I, el protocolo se considera como investigación sin riesgo. Y según la Norma que establece las disposiciones para la investigación en salud en el IMSS, «el personal que realiza actividades de investigación en salud en el IMSS deberán de efectuarlas con apego a los códigos de ética nacionales e internacionales».

\section{Resultados}

Se entregaron 108 cuestionarios (Anexo 1) y se recibieron 90 , el total de la muestra, de los que un $56.7 \%$ fueron profesores ayudantes, un $24.4 \%$ adjuntos y solo un $18.9 \%$ profesores titulares. En cuanto a la especialidad médica, las que en mayor número participaron fueron medicina interna, con un $12.2 \%$, pediatría médica con un $8.9 \%$, otorrinolaringología con un $7.8 \%$, cardiología pediátrica con un $6.7 \%$ y neumología con un $5.6 \%$ (Tabla 1 ).

Con respecto a la pregunta sobre las actividades de enseñanza que más realiza el profesor, estas fueron: organizar a los alumnos a exponer temas, el $52.2 \%$ respondió que siempre, seguida de realizar preguntas sobre temas vistos anteriormente, con un $44.4 \%$ que siempre lo hacían. De esta misma pregunta, la opción «nunca» se observó con mayor frecuencia en el dictado, con un $82.2 \%$, seguido de solicitar a los alumnos discutir temas anteriormente expuestos por el profesor, con un $43.3 \%$.

En la pregunta referente a estrategias educativas que los profesores utilizan, la opción «siempre» más frecuente fue el análisis de casos con un $55.6 \%$, seguido del aprendizaje basado en problemas con un

Tabla 1. Médicos participantes por especialidad

\begin{tabular}{|c|c|c|}
\hline Especialidad & $\mathrm{N} .^{\circ}$ & $\%$ \\
\hline Medicina interna & 11 & 12.2 \\
\hline Pediatría médica & 8 & 8.9 \\
\hline Comunicación audiología & 7 & 7.7 \\
\hline \multicolumn{3}{|l|}{ Otoneurología } \\
\hline Otorrinolaringología & 7 & 7.8 \\
\hline Cardiología pediátrica & 6 & 6.7 \\
\hline Neumología & 5 & 5.6 \\
\hline Anestesiología & 4 & 4.4 \\
\hline Reumatología & 4 & 4.4 \\
\hline Reumatología pediátrica & 3 & 3.3 \\
\hline Anatomía patológica & 2 & 2.2 \\
\hline Dermatología & 2 & 2.2 \\
\hline Genética médica & 2 & 2.2 \\
\hline Medicina crítica & 2 & 2.2 \\
\hline Neonatología & 2 & 2.2 \\
\hline Neurología & 2 & 2.2 \\
\hline Oftalmología & 2 & 2.2 \\
\hline Patología clínica & 2 & 2.2 \\
\hline Urología & 2 & 2.2 \\
\hline Otras especialidades clínicas & 17 & 18.9 \\
\hline
\end{tabular}


$42.2 \%$ y en tercer lugar las ilustraciones con un $15.6 \%$. En esta misma pregunta, la frecuencia de «nunca» que más se presento fue la simulación con un $41.1 \%$, los resúmenes con un $34.4 \%$ y los organizadores previos con un $25.6 \%$.

En relación a las respuestas para la pregunta referente a la evaluación del desempeño de los alumnos, se encontró que los métodos de evaluación más utilizados son la participación en clase (65.6\%), la exposición de temas por los alumnos (57.8\%) y los exámenes (46.7\%). Entre las estrategias de evaluación que nunca son utilizadas se encontraron la resolución de guías de estudio (45.6\%), los trabajos extra de clase $(26.7 \%)$ y los mapas conceptuales (16.7\%) (Tabla 2).

\section{Discusión}

Las actividades de enseñanza son un elemento fundamental del proceso de aprendizaje. La formación del médico especialista exige adquirir ciertas habilidades acordes a la especialidad, que se logran con la utilización y el aprovechamiento en principio de sus procesos mentales, los hábitos y actitudes adquiridas previamente para el estudio, además de adaptarse a lo largo de la especialidad al estilo de aprendizaje y enseñanza del profesor ${ }^{14,15}$.

En este estudio, como otros autores, se identificó una amplia variación entre los estilos de enseñanza; ante esto, es posible encontrar heterogeneidad en la calidad de la enseñanza ${ }^{16}$. Debemos identificar la importancia de equilibrar la relación entre lo que se enseña y cómo lo aprenden los médicos, es decir, el contenido en contraste con el proceso. De esta manera resulta importante identificar las estrategias de aprendizaje que predominan para el grupo de médicos, tanto de alumnos como de profesores ${ }^{17}$.

En el trabajo realizado encontramos que la mayoría de los profesores organiza a los alumnos para que preparen y expongan temas, y que realizan preguntas de temas vistos.

Entre las estrategias educativas señaladas como «siempre» y "casi siempre» están los mapas conceptuales, los cuadros sinópticos y los diagramas. Destacaron el análisis de casos clínicos y el aprendizaje basado en problemas, con lo que se puede identificar que los resultados de esta estrategia serán el producto de la experiencia adquirida en los años de formación previa, donde pueda aplicarse la teoría en el campo clínico, haciendo una suma de conocimientos sobre los precedentes a través de actividades como presentar y desarrollar temas del programa. Así, en las sesiones con casos clínicos resulta mejor perfilado el análisis de aquellos residentes con mayor número de años de estudio en la especialidad.

Para este estudio se recurrió al instrumento utilizado por Espinoza, et al. ${ }^{13}$, quienes reportaron, a diferencia de este trabajo, que en su estudio los profesores prefirieron exponer temas y hacer preguntas sobre temas vistos, y entre sus estrategias de enseñanza-aprendizaje utilizan demostraciones, ilustraciones y análisis de casos; respecto a la evaluación, eligen la participación en clase y los exámenes de opción múltiple.

Otros autores enfatizan que las estrategias, por sí mismas, no conducen directamente al éxito académico, sino que es condición necesaria e influyente la motivación con que el estudiante elige su aprendizaje, así como la actitud académica del docente ${ }^{18,19}$.

Es primordial que el alumno reflexione la información obtenida, que la someta a crítica; no es suficiente que adquiera la información, pues su interpretación nos permite identificar el proceso de enseñanza-aprendizaje y la evaluación constituida como un elemento integrante de este proceso ${ }^{18}$. La realización de pruebas de conocimiento y la retroalimentación individual han ayudado a los estudiantes de medicina en su ciclo de autorregulación y al docente para llevar a cabo la evaluación de todo el proceso educativo, a fin de modificar, de ser necesario, de forma dinámica las estrategias utilizadas ${ }^{20-22}$.

Los autores de este estudio identificaron que la evaluación se circunscribió a valorar el dominio de los conocimientos específicos de la especialidad. En contraste, en la evaluación por competencias, además de considerar este dominio, también se justiprecia el desarrollo del desempeño en las dimensiones cognoscitiva, actitudinal y afectivo-motivacional, para lo que se ha propuesto en principio definir la competencia entre lo que se ha aprendido y lo que se puede hacer ${ }^{12,23}$.

Así, en el enlace entre el docente y la participación del alumno, este último, para realizar una especialidad médica requiere ser ya profesional de la salud con una licenciatura, con sus propias estrategias de aprendizaje, y deberá adaptarse a las estrategias educativas del profesor. Tampoco podemos excluir que los estudiantes de las actuales generaciones utilizan herramientas tecnológicas que influyen de forma significativa en su conocimiento, al menos en el aprendizaje inmediato. De esta forma, es importante enlazar el acceso y las habilidades tecnológicas que los alumnos tienen con la estrategia educativa, con el estilo de enseñanza del profesor, facilitando el proceso de 
aprendizaje y actuando como modelo a seguir ${ }^{24}$. No basta con pensar que estamos haciendo bien las cosas, sino que hay que ponerlas a prueba. Aceptar las limitaciones en los resultados es el primer paso para definir estrategias que mejoren el proceso educativo, el refinamiento clínico de los alumnos, la seguridad del paciente y la calidad de la atención médica. Este cambio parece asociarse a la formación docente avanzada, y muy posiblemente esa fortaleza sea el camino para mejorar los procesos educativos en el posgrado. Y es que, dentro del ámbito de la medicina, los investigadores han reconocido la importancia de la autorregulación para la práctica clínica efectiva ${ }^{22,25,26}$. Con una visión participativa, favoreciendo la motivación de

Tabla 2. Métodos de enseñanza-aprendizaje utilizados por profesores médicos

\begin{tabular}{|c|c|c|c|c|}
\hline $\begin{array}{l}\text { En las asignaturas que imparte, indique con que } \\
\text { frequencia: }\end{array}$ & $\begin{array}{l}\text { Nunca } \\
\text { N. }{ }^{\circ}(\%)\end{array}$ & $\begin{array}{l}\text { Casi nunca } \\
\text { N. }{ }^{\circ}(\%)\end{array}$ & $\begin{array}{l}\text { Casi siempre } \\
\text { N. }^{\circ}(\%)\end{array}$ & $\begin{array}{l}\text { Siempre } \\
\left.\text { N. } .^{\circ} \%\right)\end{array}$ \\
\hline \multicolumn{5}{|l|}{ Realiza las siguientes actividades } \\
\hline Expongo temas & $21(23.3)$ & $30(42.2)$ & $26(28.9)$ & $4(4.4)$ \\
\hline Organizo a los alumnos para exponer temas & $1(1.1)$ & $7(7.8)$ & $35(38.9)$ & $47(52.2)$ \\
\hline Dicto & $74(82.2)$ & $13(14.4)$ & $1(1.1)$ & $2(2.2)$ \\
\hline Organizo dinámicas de grupo & $6(6.7)$ & $18(20)$ & $45(50)$ & $20(22.2)$ \\
\hline Realizo preguntas sobre temas vistos anteriormente & $2(2.2)$ & $4(4.4)$ & $41(45.6)$ & $40(44.4)$ \\
\hline $\begin{array}{l}\text { Solicito a los alumnos discutir temas expuestos } \\
\text { anteriormente por mí }\end{array}$ & $39(43.3)$ & $13(14.4)$ & $21(23.3)$ & $15(16.7)$ \\
\hline $\begin{array}{l}\text { Pido a los alumnos discutan con base en lecturas } \\
\text { previas }\end{array}$ & $12(13.3)$ & $9(10)$ & $42(46.7)$ & $27(30)$ \\
\hline \multicolumn{5}{|l|}{ ¿Utiliza las siguientes estrategias de enseñanza? } \\
\hline Organizadores previos & $23(25.6)$ & $32(35.6)$ & $28(31.1)$ & $7(7.8)$ \\
\hline Resúmenes & $31(34.4)$ & $34(37.8)$ & $20(22.2)$ & $5(5.6)$ \\
\hline Mapas conceptuales & $14(15.6)$ & $19(21.1)$ & $47(52.2)$ & $9(10)$ \\
\hline Ilustraciones & $17(18.9)$ & $28(31.1)$ & $31(34.4)$ & $14(15.6)$ \\
\hline Diagramas & $16(17.8)$ & $23(25.6)$ & $40(44.4)$ & $11(12.2)$ \\
\hline Cuadros sinópticos & $15(16.7)$ & $29(32.2)$ & $36(40)$ & $10(11.1)$ \\
\hline Analogías & $22(24.4)$ & $27(30)$ & $30(33.3)$ & $9(10)$ \\
\hline Demostraciones & $17(18.9)$ & $25(27.8)$ & $31(34.4)$ & $16(17.8)$ \\
\hline Aprendizaje basado en problemas & $2(2.2)$ & $3(3.3)$ & $47(52.2)$ & $38(42.2)$ \\
\hline Análisis de casos & 0 & $1(1.1)$ & $38(42.2)$ & $50(55.6)$ \\
\hline Simulación & $37(41.1)$ & $26(28.9)$ & $20(22.2)$ & $7(7.8)$ \\
\hline \multicolumn{5}{|l|}{$\begin{array}{l}\text { ¿Para evaluar el desempeño de sus alumnos } \\
\text { en aspectos teóricos, emplea los siguientes } \\
\text { procedimientos? }\end{array}$} \\
\hline Trabajos extra clase & $24(26.7)$ & $35(38.9)$ & $22(24.4)$ & $9(10)$ \\
\hline Portafolios de evidencias & $14(15.6)$ & $25(27.8)$ & $34(37.8)$ & $16(17.8)$ \\
\hline Exámenes & 0 & $14(15.6)$ & $34(37.8)$ & $42(46.7)$ \\
\hline Mapas conceptuales & $15(16.7)$ & $19(21.1)$ & $45(50)$ & $11(12.2)$ \\
\hline Exposiciones de temas por los alumnos & 0 & $4(4.4)$ & $34(37.8)$ & $52(57.8)$ \\
\hline Participación en clase & 0 & $2(2.2)$ & $28(31.1)$ & $59(65.6)$ \\
\hline Resolución de guías de estudio & $41(45.6)$ & $31(34.4)$ & $9(10)$ & $8(8.9)$ \\
\hline
\end{tabular}


los educandos, se busca desencadenar y encauzar la iniciativa y la inventiva promoviendo una experiencia mediada por la crítica en la elaboración del conocimiento; es un proceso donde tanto el profesor como el alumno interaccionan, se enriquecen, elaboran y transforman el conocimiento.

Un aspecto identificado como debilidad de este estudio fue que entre los profesores de las especialidades médicas que participaron en este estudio predominaron los del área clínica sobre los quirúrgicos. Con los resultados obtenidos, se interpretó que dan más importancia a la práctica con el análisis de casos clínicos, identificado como estrategia educativa sobre las actividades como realizar preguntas en relación con temas vistos anteriormente.

En este siglo XXI queda otro reto por resolver: plantear algunas consideraciones para la reflexión y alternativas de acción en torno a la didáctica en las especialidades médicas. Ante la creciente preocupación por la seguridad del paciente en el ámbito hospitalario han surgido cuestionamientos sobre cómo se adquiere la eficacia de la experiencia práctica de los profesionales de la salud. Al presente existe la educación por simulación mediante equipos tecnológicos con metodologías educativas que han probado ser eficientes ${ }^{27}$, sin que por ello no exista la interacción de profesor y alumno, promoviendo la elaboración del conocimiento.

\section{Agradecimientos}

A todos los profesores médicos que contribuyeron con su tiempo y sus respuestas para este estudio.

\section{Financiamiento}

Este estudio no requirió financiamiento.

\section{Bibliografía}

1. Chan D. Validation of the clinical learning environment inventory. Western Journal of Nursing Research. 2003;5:519-32.

2. Palacios GM, Quiroga LP. Percepción de los estudiantes de las características y comportamientos de sus profesores asociados a una enseñanza clínica efectiva. Estudios Pedagógicos. 2012;1:73-87.

3. Martínez GP. Aprender y enseñar: los estilos de aprendizaje y de enseñanza desde la práctica del aula. Bilbao, España: Mensajero; 2008.

4. Zhang L, Sternberg RJ. The nature of intellectual styles. $2^{\text {nd }}$ ed. USA: Education 2012.
5. Díaz-Barriga AF, Hernández RG. Estrategias docentes para un aprendizaje significativo. Una interpretación constructivista. $2^{a}$ ed. México: McGraw Hill; 2002. Citado en: Palacios GM, Quiroga LP. Percepción de los estudiantes de las características y comportamientos de sus profesores asociados a una enseñanza clínica efectiva. Estudios Pedagógicos. 2012;1:73-87.

6. Stein GE. Eficacia docente en la educación odontológica. (Consultado el 10 de mayo de 2015.) Disponible en: http://www.odontologiaactual. com/eficacia-docente-en-la-educacion-odontologica.

7. Bello BS. Elementos a considerar por el docente clínico en odontología para la elaboración de estrategias de enseñanza clínica. Ciencia Odontológica. 2012;9:112-22.

8. García-Jiménez EP, Rojas-Pérez EM, Ruiz-Ruisanchez A. Técnicas de estudio para mejorar el aprendizaje en la residencia médica. Rev Mex Anestesiología. 2012;35:S242-4.

9. Arias CM, Cano PE, Torres LJ. Estrategias de aprendizaje de los residentes en medicina general integral del Centro Oftalmológico José Martí. Educ Med Sup. 2010;24:223-39.

10. Aguilera PE. Los estilos de enseñanza, una necesidad para la atención de los estilos de aprendizaje en la educación universitaria. Journal of Learning Styles. 2012;10:79-87. (Consultado en octubre de 2015.) Disponible en: https://dialnet.unirioja.es/revista/12944/V/10

11. Sánchez SA, Cisterna CF. La evaluación de los aprendizajes orientada al desarrollo de competencias en odontología. Educ Med Sup. 2014;28:104-14.

12. McDonald R, Boud D, Francis J, et al. Nuevas perspectivas sobre la evaluación. Boletín Cinterfor. 2000;149:41-72. (Consultado en abril de 2015.) Disponible en: http://www.oitcinterfor.org/sites/default/files/file_articulo/rodajog.pdf.

13. Espinoza-Vázquez O, Martínez-González A, Díaz-Barriga F. Formas de enseñanza y evaluación utilizadas por los docentes de odontología: resultados y su clasificación psicopedagógica. Inv Edu Med. 2013;2:183-92.

14. Lifshitz A. La enseñanza de la competencia clínica. Gac Med Mex. 2004;140:312-3.

15. Gaona-Flores VA, Campos-Navarro LA, Alcalá-Martínez E. Transformative learning between the student and the teacher? Concerning the learning styles of medical residents. Global Advanced Research Journal of Medicine and Medical Science GATMMMS. 2014;31:445-50.

16. Cid CS. El uso de estrategias de aprendizaje y su correlación con la motivación de logro en los estudiantes. REICE. 2008;6:100-20.

17. Estrada-Valenzuela CM, Prado-Mendoza JJ, De la Fuente-Cabrera LP, et al. Eficacia de estrategias de aprendizaje utilizadas por alumnos de la Facultad de Odontología de la Universidad Autónoma de Coahuila. Unidad Torreón. Rev Tamé. 2014;3:264-70.

18. Viniegra-Velázquez L. El progreso y la educación En: Educación y crítica. El proceso de elaboración del conocimiento. México: Editorial Paidós Mexicana; 2002. p. 83-98.

19. Chiecher AC, Donolo DS, Corica JL. Entornos virtuales de aprendizaje: nuevas perspectivas de estudio e investigaciones. Mendoza: Editorial Virtual Argentina; 2013.

20. Chang A, Chou CL, Teherani A, et al. Clinical skills-related learning goals of senior medical students after performance feedback. Med Educ. 2011;45:878-885. (Consultado el 25 de enero de 2016.) Disponible en: http://onlinelibrary.wiley.com/doi/10.1111/j.1365-2923.2011.04015.x/epdf.

21. Viniegra-Velázquez L. El desafío de la educación médica en el Instituto Mexicano del Seguro Social. Cómo constituirse en la avanzada de la superación institucional. Rev Med Inst Mex Seguro Soc. 2005;43:305-21.

22. Sandars J, Cleary TJ. Self-regulation theory: applications to medical education: AMEE Guide No. 58. Medical Teacher. 2011;33:875-86.

23. González-López E, García-Lázaro I, Blanco-Alfonso A, et al. Aprendizaje basado en la resolución de problemas: una experiencia práctica. Educ Med. 2010;13:15-24

24. Spencer J. ABC of learning and teaching in medicine. BMJ. 2003; 326:591-4.

25. Brydges R, Peets A, Issenberg B, et al. Divergence in student and educator conceptual structures during auscultation training. Med Educ. 2013;47:198-209.

26. Sandars J. When I say self-regulated learning. Med Educ. 2013;47:1162-3.

27. Wayane DB, Didwania A, Feinglass J, et al. Simulation based education improves quality of care during cardiac arrest team responses at an academic teaching hospital. American College of Chest Physicians. Chest. 2008;133:156-61. 
Anexo 1. Cuestionario sobre la identificación de estrategias educativas y evaluación.

Marque con $\mathrm{X}$ su respuesta en el recuadro correspondiente a cada pregunta

Nombramiento

Profesor titular

Profesor adjunto

Profesor ayudante

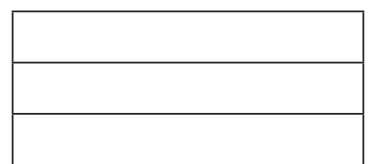

Antigüedad como profesor

1-5 años

5-10 años

11-15 años

15-20 años

Más de 20 años

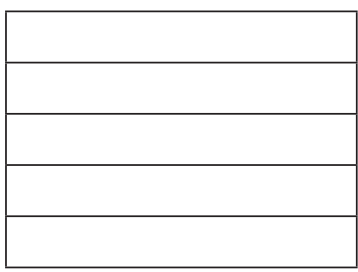

Especialidad

En las asignaturas que imparte, indique con qué frecuencia realiza las siguientes actividades
a) Expongo temas
b) Organizo a los alumnos para que expongan temas
c) Dicto
c) Organizo dinámicas de grupo
d) Realizo preguntas sobre temas vistos anteriormente
e) Solicito a los alumnos discutan acerca de lo expuesto por mí
f) Pido a los alumnos que discutan con base en lecturas previas

¿Con qué frecuencia utiliza las siguientes estrategias de enseñanza en las asignaturas que imparte?
a) Organizadores previos
b) Resúmenes
c) Mapas conceptuales
d) Ilustraciones
e) Diagramas
f) Cuadros Sinópticos
g) Analogías
h) Demostraciones
i) Aprendizaje basado en problemas
j) Análisis de casos
k) Simulación

Para evaluar el desempeño de sus alumnos en los aspectos teóricos, señale con qué frecuencia emplea los siguientes procedimientos
a) Trabajos extra clase
b) Portafolios de evidencias
c) Exámenes
d) Mapas conceptuales
e) Exposicones de temas por los alumnos
f) Paiticipación en clase
g) Resolución de guías de estudio

\begin{tabular}{|l|l|l|l|}
\hline Nunca & Casi nunca & Casi siempre & Siempre \\
\hline & & & \\
\hline & & & \\
\hline & & & \\
\hline & & & \\
\hline & & & \\
\hline & & & \\
\hline & & & \\
\hline
\end{tabular}

\begin{tabular}{|l|l|l|l|}
\hline Nunca & Casi nunca & Casi siempre & Siempre \\
\hline & & & \\
\hline & & & \\
\hline & & & \\
\hline & & & \\
\hline & & & \\
\hline & & & \\
\hline & & & \\
\hline & & & \\
\hline & & & \\
\hline & & & \\
\hline & & & \\
\hline
\end{tabular}

\begin{tabular}{|l|l|l|l|}
\hline Nunca & Casi nunca & Casi siempre & Siempre \\
\hline & & & \\
\hline & & & \\
\hline & & & \\
\hline & & & \\
\hline & & & \\
\hline & & & \\
\hline
\end{tabular}

